\title{
Sammelbesprechung
}

\section{Renaissance eines „Gespenstes“? Aktuelle Auseinandersetzungen mit den Theorien von Karl Marx}

Martin Beck / Ingo Stützle (Hrsg.), Die neuen Bonapartisten. Mit Marx den Aufstieg von Trump \& Co. verstehen. Berlin: Dietz 2018, 272 S., br., 18,00€

Tine Haubner / Tilman Reitz (Hrsg.), Marxismus und Soziologie. Klassenherrschaft, Ideologie und kapitalistische Krisendynamik. Weinheim/Basel: Beltz Juventa 2018, 276 S., br., 29,95€

Stefan Kühl, Arbeit - Marxistische und systemtheoretische Zugänge. Wiesbaden: Springer VS 2018, 139 S, kt., 17,99€

Rainer Lucas / Reinhard Pfriem / Claus Thomasberger (Hrsg.), Auf der Suche nach dem Ökonomischen - Karl Marx zum 200. Geburtstag. Marburg: Metropolis 2018, 492 S., gb., 34,80€

Alexandra Scheele / Stefanie Wöhl (Hrsg.), Feminismus und Marxismus. Weinheim/Basel: Beltz Juventa 2018, 250 S., br., 29,95€

Ingo Schmidt / Carlo Fanelli (Eds.), Reading 'Capital Today'. Marx after 150 Years. London: Pluto Press 2017, 224 S., kt., 13,99€

Besprochen von Assoz. Prof. Dr. Roland Atzmüller: Abteilung für Gesellschaftstheorie und Sozialanalysen, Institut für Soziologie, JKU Linz/Österreich, E-Mail: Roland.Atzmueller@jku.at

https://doi.org/10.1515/srsr-2020-0069

Schlüsselwörter: Krise des Marxismus, Entwicklungen marxistischer Theoriebildung und Wissenschaft, Transformation kapitalistischer Gesellschaften, feministische Kritik des Marxismus

\section{Einleitung}

In den letzten Jahren sind, rund um die Marx-bezogenen Jubiläen (200. Geburtstag, 150 Jahre „Das Kapital“), eine Reihe von Publikationen erschienen, die die Theorien von Marx und die daran anknüpfenden Debatten auf den Prüfstand sozialwissenschaftlicher Debatten stellen. Dazu gehören einerseits Publikationen, die an der Aktualisierung und Weiterentwicklung marx(isti)scher Theoriebildung zu arbeiten beanspruchen und die marx(isti)sche Konzepte für die Untersuchung 
aktueller gesellschaftlicher Phänomene fruchtbar machen wollen. Andererseits sind auch Arbeiten erschienen, die marx(isti)sche Ansätze vor dem Hintergrund alternativer sozialwissenschaftlicher Theorietraditionen (z.B. Differenzierungstheorie/Systemtheorie) diskutieren. Solche Arbeiten können zur Schärfung der verschiedenen Positionen beitragen, sie zeigen in ihren Qualitäten aber auch die aktuelle wahrheitspolitische Bedeutung der marxistischen Theoriebildung in den Sozialwissenschaften und inwiefern diese anderen Ansätzen eine adäquate Auseinandersetzung abverlangt.

Die verschiedenen Publikationen deuten darauf hin, dass in den Sozialwissenschaften, aber auch in öffentlichen Debatten spätestens seit der Finanzkrise 2008, der darauf folgenden Staatsschuldenkrise und ihrer austeritätspolitischen Bearbeitung wieder ein „Gespenst“ (Marx/Engels, 1959a: 461) umgeht - nämlich die Theorien von Karl Marx. Aber gerade in den öffentlichen, teilweise auch in den sozialwissenschaftlichen Auseinandersetzungen erweist sich das Herumgeistern des Marxschen Gespenstes oft als geschichtslos und hochgradig selektiv. Die Auseinandersetzung mit marx(isti)schen Theorien und Debatten geht mit wenig Bezugnahme auf ihre historischen Entwicklungen vonstatten und es überwiegt eine reduktionistische Perspektive auf einen Lehrbuchmarx, die oft eher an seine marxistisch-lenistische Kanonisierung erinnert, die hier unter der Hand perpetuiert wird. Die Frage nach den historischen Entwicklungen verschiedener Rezeptionsstrategien der Marxschen Konzepte und Begriffe sowie ihrer permanent notwendigen Aktualisierung und sich ändernden Artikulation in den sozialen Auseinandersetzungen wird ausgeblendet, sodass Marx zwar als wichtiger, von den gesellschaftlichen Entwicklungen aber überholter Denker des 19. Jahrhunderts historisiert werden kann. Die marxistischen Theorietraditionen werden so als veraltete und daher unterkomplexe Herangehensweise an die Auseinandersetzung mit aktuellen gesellschaftlichen Entwicklungen dargestellt. Diese Historisierung von Marx ermöglicht in weiterer Folge die selektive Rezeption Marxscher Begriffe und Konzepte, was aus dem Marxschen Theorieprojekt bloße Komponenten eines wie auch immer gefassten „Werkzeugkastens“ (Michel Foucault) der Sozialwissenschaften macht, aus dem man sich im marx(isti)schen Forschungsprozess selektiv bedienen könne. Damit aber werden dem marxistischen Theorieprojekt wesentliche Momente seines Erkenntnisanspruches genommen, der erstens darin besteht, Begriffe und Konzepte zu entwickeln, die die kritische Analyse moderner Gesellschaften in ihrem Gesamtzusammenhang als komplexes Ganzes, also in ihrer Totalität, ermöglichen sollen. Das bedeutet übrigens nicht, um eine Kritik von Ingrid Kurz-Scherf (in Scheele/Wöhl: 59f.) vorwegzunehmen, dass dies in den historischen Theoriedebatten des Marxismus je vollständig geleistet hätte werden können. Vielmehr ist davon auszugehen, dass auch kritische Analysen des gesellschaftlichen Gesamtzusammenhanges nicht nur stets unvollständig, sondern im- 
mer auch temporär sind, da sie von den gesellschaftlichen Entwicklungen permanent überholt werden (Atzmüller, 2018). Zweitens verweist der skizzierte Erkenntnisanspruch auf das Praxisproblem des Marxismus als kritischer Gesellschaftstheorie (Kurz-Scherf in Scheele/Wöhl: 63), das aus der Perspektive des marxistischen Theorieprojektes ja darin besteht, dass es nicht nur darauf ankommt, die Welt unterschiedlich zu analysieren, sondern (es möglich ist,) sie zu verändern (siehe dazu: Marx/Engels, 1959b). Ein Zusammenhang, der aber unter der gegenwärtigen gesellschaftlichen Konstellation zerrissen ist.

Um den „Stand des Marxismus“ (Demirovic et al., 2015) in den sozialwissenschaftlichen Debatten und sein analytisches Potenzial für die Untersuchung der Gegenwartsgesellschaft einschätzen zu können, ist erstens interessant, in welcher Tiefe und Ausdifferenziertheit sich alternative sozialwissenschaftliche Ansätze mit den gegenwärtigen Entwicklungen marxistischer Theorien und Forschungsstrategien auseinandersetzen (II.). Zweitens stellt sich die Frage, wie in den sozialwissenschaftlichen Debatten (III.) das analytische Potenzial der marxistischen Theorietraditionen weiter zu entwickeln versucht wird. Dies betrifft nicht nur die innermarxistischen Debatten, sondern v.a. auch die Rolle des „marxistischen Denkens“ (Haubner/Reitz) in der Soziologie sowie auch sein Verhältnis zu feministischen Theorietraditionen bzw. den Anspruch einer disziplinenübergreifenden und interdisziplinären Analyse der Gesellschaften (Lucas et al.), in denen die kapitalistische Produktionsweise herrscht (Marx, 1972: 49). Darüber hinaus geht es jenseits solcher grundlegender Überlegungen immer auch darum, inwiefern spezifische theoretische Überlegungen von Marx zur Analyse aktueller Probleme (Rechtsentwicklung in vielen Ländern und neuer Autoritarismus) beitragen können (IV.).

\section{Systemtheoretische Umwälzungen des Marxismus}

Wie eingangs angedeutet, zeigt sich die aktuelle Relevanz des Marxismus auch daran, ob andere gesellschaftstheoretische Ansätze sich ernsthaft mit seinen wesentlichen Kategorien wie auch konkreten Analysen auseinandersetzen. Ein Beispiel dafür stellt die Untersuchung „Arbeit - Marxistische und systemtheoretische Zugänge" von Stefan Kühl dar. Kühl diskutiert in diesem Band die marxistischen wie auch systemtheoretischen Bestimmungen und Untersuchungen zum Thema Arbeit und ihre Bedeutung für moderne Gesellschaften, Organisationen und Bewusstseinsformen und versteht dies als Antwort auf die gesellschaftstheoretische Abstinenz der Sozialwissenschaften oder deren Beschränkung auf Theorien mitt- 
lerer Reichweite seit den 1990er Jahren (4). Die Kühlsche Rezeption der marx(isti) schen Theorien beruht dabei aber immer wieder auf Fehlinterpretationen und Verkürzungen, wie sie eingangs angesprochen wurden. Diese beeinflussen auch seine Kritikstrategie gegenüber marxistischen Ansätzen. So nimmt Kühl etwa auf die Marxschen Begriffe zur Untersuchung der Ware Arbeitskraft Bezug (15ff.). Er setzt dabei, richtigerweise, an ihrem Doppelcharakter an. Dieser besteht darin, Gebrauchswert und Tauschwert zu sein, was Marx als „Springpunkt“ bezeichnete, „um den sich das Verständnis der politischen Ökonomie dreht“ (Marx, 1972: 56). Aus der Sicht der Arbeitskräfte besteht der Gebrauchswert ihrer Arbeitskraft in der konkreten, nützlichen Arbeit, die sie verrichten können. Der Tauschwert ihrer Arbeit, den die Arbeitskräfte als Lohn erhalten, ist unter den Bedingungen der kapitalistischen Produktionsverhältnisse wesentlich durch die (aus verschiedenen Gründen schwankenden und umkämpften) Reproduktionskosten für ihr Überleben (und das ihrer Familie) bestimmt. Wichtig ist nun, dass aus der Sicht des Kapitals der Gebrauchswert der Ware Arbeitskraft darin besteht, dass sie - im Unterschied zu anderen Waren - im Produktionsprozess einen höheren Wert (also Mehrwert) als den zu ihrer Reproduktion notwendigen produzieren kann. Diese Unterscheidung vermischt Kühl jedoch, was z.B. in folgender Feststellung sichtbar wird: „Der springende Punkt ist nun - und das ist der Kern der Marx'schen Kapitalismustheorie - dass der Gebrauchswert der Arbeit größer ist als ihr Tauschwert“ (Kühl: 15).

Tauschwert und Gebrauchswert sind jedoch qualitativ unterschieden und können daher nicht in eine quantitativ bestimmte (,größer“) Beziehung zueinander gebracht werden. Durch dieses Missverständnis entgeht Kühl aber der weitreichende Gehalt der Marxschen Überlegungen zum Doppelcharakter der Arbeit, die es ermöglichen, die Arbeitsteilungen und Produktionsverhältnisse im Kapitalismus als Ausbeutungs- und Herrschaftsverhältnisse zu erfassen und darin auch zentrale Triebkräfte der Entwicklung der (warenförmigen) Arbeit (z.B. Dequalifikation und Entwertung $)^{1}$ zu erkennen. Eine derartige Perspektive kann nicht auf das Problem von Hierarchien in Organisationen reduziert werden, wie Kühl es marxistischen Ansätzen zuschreibt.

Er gewinnt aber aus derart missverständlichen Interpretationen der marxistischen Theorien wesentliche Dimensionen seiner Argumentationsstrategie. Diese besteht darin, den nach seiner Interpretation „kausal relativ simplen Annahmen über die Auswirkung der Produktionsverhältnisse“ (Kühl: 118) im Marxismus eine differenzierungstheoretische Herangehensweise entgegen zu setzen, die ein komplexeres Bild von Gesellschaft ermögliche. Die Komplexität differenzierungstheo-

1 Dazu gibt es aber immer auch Gegentendenzen. 
retischer Interpretationen sozialer Prozesse bestimmt Kühl dann ,in der am weitesten gehenden Form der funktionalen Differenzierung“ (Kühl: 32) so, dass „gesellschaftliche Teilbereiche nur noch nach einfachen Zweier-Schemata (funktionieren): In der Wirtschaft interessiert nur noch, ob gezahlt wird oder ob nicht gezahlt wird und nicht, ob einem die Hautfarbe, das Geschlecht oder die politische Orientierung eines Kunden oder Verkäufers genehm“ ist (ebd.).

Nun ist damit erstens noch kein spezifischer Erkenntnisgewinn der Systemtheorie gegenüber dem Marxismus benannt, da schon Marx und Engels im kommunistischen Manifest herausgearbeitet hatten, dass der Kapitalismus „kein anderes Band zwischen Mensch und Mensch übriggelassen [habe] als das nackte Interesse, als die gefühllose ,bare Zahlung““ (Marx/Engels, 1959a: 464). Die Differenz zwischen aktuellen (nicht zuletzt) an Marx orientierten Debatten und einer derart interpretierten Systemtheorie besteht aber darin, dass erstere nicht nur die wirtschaftlichen Interaktionen in ihrer widersprüchlichen Herrschaftsförmigkeit und ihren Auswirkungen auf Arbeit verstehen wollen, sondern auch die Frage ins Zentrum rücken, wie diese etwa durch rassistische und geschlechtsspezifische Dynamiken überformt sind. Darauf geht Kühl aber nicht ein. Dies ist in gewisser Weise paradigmatisch für seine Argumentationsstrategie, die keine Notwendigkeit sieht, in seiner Darstellung der systemtheoretischen Perspektive auf (mögliche oder gängige) Gegenargumente oder Anti-Kritiken, wie sie eben auch von marxistischer Seite immer wieder vorgebracht wurden (Demirović, 2001), einzugehen. Insgesamt beruht daher diese hier exemplarisch dargestellte Herangehensweise an marx(isti)sche Debatten auf einer verengten und vereinseitigten Interpretation letzterer, der sicher in der Geschichte marxistischer Theoriebildung oftmals Vorschub geleistet wurde.

Sie ignoriert damit aber, dass in den selbstreflexiven Debatten des Marxismus der letzten Jahre versucht wurde, derartige Dogmatisierungen zu überwinden und den offenen und unabgeschlossenen Charakter des Marxschen Theorieprojektes herauszustreichen, der nicht nur aus der Begrenztheit der wissenschaftlichen Leistungen einer einzelnen Person herrührt, sondern auch auf die grundlegende Veränderbarkeit kapitalistischer Gesellschaftsformationen zurückzuführen ist, die eine permanente Revision der theoretischen Grundlagen erzwingt. 


\section{IIla. Herausforderungen für eine Erneuerung marxistischen Denkens zwischen Selbstreflexion und Praxisproblem}

Eine Aktualisierung marxistischer Theoriebildung muss daher erstens an der Entwicklungsfähigkeit der kapitalistischen Produktionsweise ansetzen, die zum Ausgangspunkt ihrer kritischen Weiterentwicklung gemacht werden muss und auch als Voraussetzung für ihre „Praxisrelevanz“ (Kurz-Scherf in Scheele/Wöhl) anzusehen ist (siehe insbesondere Schmidt/Fanelli; Haubner/Reitz). Zweitens muss ihre Aktualisierung Leerstellen und Lücken in den Blick nehmen sowie begriffliche Werkzeuge entwickeln, die die Analyse gesellschaftlicher Bereiche ermöglichen und die über die Kritik der politischen Ökonomie im engeren bzw. verengten Sinne hinausgehen, wie dies (paradigmatisch) etwa in den feministisch-marxistischen Debatten (Federici in Schmidt/Fanelli; Scheele/Wöhl) vorangetrieben wird.

Der Band von Ingo Schmidt und Carlo Fanelli „Reading 'Capital Today“" stellt ein interessantes Beispiel dar, wie innerhalb der Theorietraditionen des westlichen Marxismus die aktuellen Herausforderungen an eine Erneuerung des marxistischen Theorieprogramms und seine Verknüpfung mit sozialen Kämpfen und politischen Aktivitäten bestimmt werden. Der Titel des Bandes paraphrasiert nicht nur eine zentrale Schrift des westlichen Marxismus, nämlich „Das Kapital lesen“ (Althusser et al., 2015), sondern verweist zugleich auf eine zentrale Fragestellung gegenwärtiger Auseinandersetzungen mit Marx. Diese besteht darin, wie die Theorien von Marx (und Engels) und insbesondere „Das Kapital“ im gegenwärtigen historischen Kontext (Schmidt/Fanelli: 2), der von weitreichenden Änderungen der kapitalistischen Produktionsverhältnisse, aber auch von Staat und Politik seit den 1970er Jahren geprägt ist, gelesen werden können. Dabei geht es nicht nur um die Entwicklung einer zeitgemäßen Analyse des Gegenwartskapitalismus, sondern auch um die sich ändernden und widersprüchlichen Zusammenhänge zwischen marxistisch inspirierter Gesellschaftskritik und sozialen Kämpfen bzw. sozialistischen Strategien. Das von Kurz-Scherf (s. o.) angesprochene Praxisproblem marxistischer Theoriebildung stellt daher den zentralen Problemhorizont der im Band von Schmidt und Fanelli gesammelten aktuellen und historischen Analysen dar. $\mathrm{Zu}$ den zentralen Themenstellungen gehören Untersuchungen zu den Veränderungen in den kapitalistischen Arbeitsprozessen (Paul Thompson und Chris Smith) sowie im Bereich der Reproduktions- und Sorgearbeit (Silvia Federici), eine marxistische fundierte Analyse zur Zerstörung der Umwelt (Hannah Holleman) sowie ein Beitrag zu den Bedingungen der Organisation der Arbeiter`innenklasse (Carlo Fanelli und Jeff Noonan). 
Es zeichnet einige der hier versammelten Aufsätze aus, dass ihre Fragestellungen auf weitreichende Auseinandersetzungen zwischen verschiedenen marxistischen Debattensträngen abzielen, wie am Aufsatz von Thompson und Smith gezeigt werden kann. Diese diskutieren den empirischen (und konzeptuellen) Gehalt der sogenannten post-operaistischen Analysen „immaterieller Arbeit“. Letztere sind wesentlich mit Namen wie Michael Hardt oder Toni Negri und ihrer Schrift Empire (2000) verbunden, die Anfang der 2000er Jahre Furore machte. Post-operaistische Ansätze gehen, vereinfacht gesprochen, davon aus, dass die kapitalistischen Arbeitsprozesse im kognitiven Kapitalismus, der sich nach dem Fordismus durchgesetzt habe, durch die Dominanz der kooperativen, kreativen, kommunikativen und kognitiven Fähigkeiten (Hardt/Negri, 2000: 284-294) der Arbeitskräfte bestimmt sind. Durch diese Verschiebungen wären zentrale Bestimmungen der marxistischen Wertheorie, die den Wert einer Ware an der aufgewendeten Arbeitszeit festmacht, überholt, da sich immaterielle Tätigkeiten der Messbarkeit in Zeiteinheiten und damit auch der Kontrolle durch das Kapital entziehen. Post-operaistische Debatten schlussfolgern daraus, dass die Rolle des Kapitals in den Produktionsverhältnissen und für die Arbeitsprozesse zunehmend „parasitic“ (Thompson/Smith: 126 in Schmidt/Fanelli) werde und dass die entstehenden netzwerkartigen Produktionsstrukturen auf eine Überwindung der kapitalistischen Produktionsverhältnisse hinauslaufen. Thompson und Smith zeigen demgegenüber unter Bezugnahme auf empirische Forschungen, dass erstens das Ausmaß der erfassten Veränderungen überschätzt wird und dass zweitens die Unternehmen auch immaterielle Arbeit einer Reihe von Kontrollmechanismen (Stichwort digitalisierter Taylorismus) unterziehen. Sie argumentieren daher, dass die Hinweise auf eine Überwindung der kapitalistischen Arbeitsteilungen durch die empirischen Erkenntnisse nicht gedeckt sind und dass damit auch die politischen Schlussfolgerungen, die darin eine Grundlage für die Überwindung des Kapitalismus durch eine zur Multitude transformierte Arbeiter`innenklasse sehen, überzogen sind. Vielmehr erschweren derartige Zuspitzungen die Auseinandersetzung mit Widerstandsmöglichkeiten gegen heterogenisierte Beschäftigungsverhältnisse und Arbeitsbedingungen.

Die aktualisierte Lektüre einer Schrift wie „Das Kapital“ kann daher, wie Schmidt und Fanelli vor dem Hintergrund dieser Analysen darlegen, keine Rückkehr zu einer kanonisierten und dogmatisierten, eigentlichen und unverstellten Marx-Interpretation sein. Vielmehr ist zu verstehen, wie sich der Kapitalismus nicht zuletzt (auch) durch die sozialen Kämpfe transformiert hat und welche Herausforderungen daraus für historisch spezifische Lesarten und Rezeptionsstrategien des „Kapitals“ und der Weiterentwicklung seiner theoretischen Begrifflichkeiten resultieren. Neue Lesarten und Rezeptionsstrategien Marxscher Theorien haben sich demnach stets in Auseinandersetzung mit gesellschaftlichen wie auch 
politischen sowie intellektuellen Krisen entwickelt (Anderson, 1978). Dadurch wurde nicht nur die theoretische und analytische Reichweite marxistischer Ansätze erweitert, sondern auch die Untersuchung aktueller gesellschaftlicher Veränderungen ermöglicht.

„Such innovation further requires incorporating issues into Marxist analysis that lay beyond the scope of Capital, notably the non-capitalist worlds of nature, household labour and subsistence production" (Schmidt: 32 in Schmidt/Fanelli).

\section{IIIb. Auseinandersetzungen mit marxistischem Denken in den Sozialwissenschaften}

Damit der Anspruch einer Aktualisierung des Marxschen Theorieprojektes geleistet werden kann, stellt sich daher die Frage, wie marxistisch orientierte Debatten sich in den gegenwärtigen sozialwissenschaftlichen Auseinandersetzungen und Disziplinen verorten, da historische Erneuerungsbewegungen des Marxismus immer auch in der kritischen Auseinandersetzung mit wissenschaftlichen Entwicklungen in verschiedenen Disziplinen geschahen.

Der von Tine Haubner und Tilman Reitz herausgegebene Band „Marxismus und Soziologie“ beansprucht zu „erproben“ (7), inwiefern das „marxistische Denken“ (ebd.) „einen Beitrag zum Verständnis der Gegenwartsgesellschaft“ (ebd.) leisten kann. In vier Themenblöcken widmen sich die Aufsätze dieses Bandes Problemstellungen, die für die Aktualisierung „marxistischen Denkens“ von grundlegender Bedeutung sind. Die zentralen Themen des Buches umfassen Auseinandersetzungen mit Veränderungen der „Klassen- und Ausbeutungsverhältnisse“, die sich bspw. mit den Grenzen des Marxschen Klassenkonzeptes (Marcel van der Linden) oder der Bedeutung des industriellen Aufstiegs Chinas aus klassentheoretischer Perspektive (Stefan Schmalz) auseinandersetzen; weiters Untersuchungen zu „Ideologien und ideologischen Apparaten“, die sich bspw. mit Religionskritik (Jan Rehman) und Kulturindustrie (Susanne Martin) befassen. Darüber hinaus wird anhand der Themenauswahl klar, dass die hier analysierten Herausforderungen sich nicht mehr auf Krisen- und Transformationstendenzen in einzelnen Nationalstaaten eingrenzen lassen, wie dies etwa Jens Wissel in seinem Aufsatz über Globale Arbeitsteilung, Nationalstaat und Migration thematisiert. Vielmehr bedrohen sie zunehmend, wie Thomas Barth für die ökologischen Krisen im Kontext des Klimawandels zeigt, die Reproduktionsfähigkeit von globalisierten Gesellschaftsformationen. Insgesamt zeigen die Aufsätze dieses Bandes also, wie die Entwicklungstendenzen kapitalistischer Gesellschaftsformationen und die daraus resultierenden kategorialen Probleme z.B. der Neubestimmung der gesell- 
schaftlichen Klassenverhältnisse mit globalen und systemischen Dynamiken verwoben sind.

Neben diesen eher auf konkrete Dynamiken fokussierten Aufsätzen ist ein Themenkomplex des Bandes außerdem an eher wissenschaftstheoretischen Fragen ausgerichtet und widmet sich Lücken der marx(isti)schen Theoriebildung etwa in der Klassentheorie und der Einbeziehung askriptiver Formen von Ungleichheit (Urs Lindner) oder der Arbeitswertlehre (Tilman Reitz). Die Analysen im Abschnitt zum Theorienstreit sind leider insgesamt am wenigsten überzeugend, da einige der Aufsätze schlicht zu viel wollen. So versucht etwa Urs Lindner die Marxsche Klassentheorie mit der Analyse von Ungleichheiten, die durch Geschlecht und Ethnizität entstehen, zu verbinden. Dabei geht er jedoch nicht ausreichend auf die Entwicklung der Debatten zur marxistischen Klassentheorie (insbesondere in den letzten Jahrzehnten) ein und fragt daher nicht, inwiefern etwa die Auseinandersetzungen um die neuen Mittelklassen, aber ebenso die feministischen und anti-rassistischen Interventionen auch Antworten jenseits der Theorie „askriptiver Ungleichheit“ bieten können.

Insgesamt sind die in diesem Band versammelten Themenschwerpunkte für eine Erprobung des „marxistischen Denkens“ zur Analyse der Gegenwartsgesellschaft zweifelsohne von zentraler Bedeutung, auch wenn sie angesichts des marxistischen Anspruchs, die Totalität kapitalistischer Gesellschaftsformationen zu erfassen, sicher nicht erschöpfend sind. So ist erstens schade, dass jüngste Debatten um die Klassenbestimmung des sogenannten Prekariats, die Entwicklung des Staates oder aber die Rolle von Rassismus für die Neuzusammensetzung der Klassenverhältnisse weitgehend ausgespart bleiben. Auch die feministische Kritik und Weiterentwicklung marxistischer Kategorien zur Analyse von Ausbeutung beschränkt sich auf den Aufsatz von Tine Haubner, die versucht, einen Ausbeutungsbegriff zu entwickeln, der nicht nur auf die Abpressung von Mehrwert angewendet werden kann, sondern auch auf die Aneignung von (unbezahlter) Careund Reproduktionsarbeit.

Zweitens wird bei der Lektüre der Aufsätze noch eine weitere Lücke dieses Bandes sichtbar, der in einer weitgehenden Ausblendung der historischen Entwicklung der an Marx orientierten Debatten zu den verschiedenen Problemen in verschiedenen Konstellation zu sehen ist. Wären die verschiedenen Aufsätze stärker in diese historischen Linien eingebettet, würde das die Stärken des vorliegenden Bandes akzentuieren. Ihr Ausblenden aber ist deswegen problematisch, weil sich der Bedeutungsverlust marxistischer Theorie und Wissenschaft an den Universitäten auch im Kappen historischer Linien manifestiert bzw. durch diese noch verstärkt wird.

So fällt schon in der Einleitung auf, dass sich die Herausgeber`innen nicht ganz sicher zu sein scheinen, wie sie das Verhältnis zwischen „marxistischem 
Denken“ und der (Gegenwarts-)Soziologie fassen sollen. Sie heben einerseits hervor, dass man das Verhältnis zwischen der akademischen Soziologie und der „revolutionären Denkschule“ (8) als gespannt bezeichnen könne und dass es von wechselseitigen Ideologievorwürfen und im Falle des Marxismus auch von seiner Gleichsetzung mit dem Marxismus-Leninismus geprägt sei. Außerdem verweisen sie unter Bezugnahme auf Niklas Luhmann (ebd.) auch auf Abwehrstrategien gegen marxistische Kritik, die diese als veraltet und überholt präsentieren (s.o.). Die Frage, wie sich die Position der marxistischen Wissenschaftstradition in den Sozialwissenschaften, die einmal, wie Haubner und Reitz unter Bezugnahme auf Ralf Dahrendorf hervorheben, für deren „Einheit“ (ebd.) stand, in den letzten Jahren verändert hat, thematisieren sie aber leider nicht. Sie betonen zwar, dass sie „glauben“ (12), dass marxistische Forschung in den „Kernbereich sozialwissenschaftlicher Forschung“ gehöre, „solange wir in kapitalistischen Verhältnissen leben“ (ebd.). Doch diese Formulierung ist irritierend, kann es doch in der Frage um die analytische Relevanz marxistischer Theoriebildung und Wissenschaft nicht darum gehen, diese auf eine Glaubensfrage zu reduzieren. Dadurch bringen sich die Herausgeber*innen in eine letztlich defensive Position, die angesichts der im vorliegenden Band angerissenen Themen gar nicht notwendig wäre.

Durchaus selbstbewusster präsentiert sich der von Alexandra Scheele und Stefanie Wöhl herausgegebene Band „Feminismus und Marxismus“, der der Frage nachgeht, „welche feministischen Herausforderungen weiter mit dem Marxismus und seiner Rezeption verbunden sind“ (8). Die Herausgeberinnen beschreiben die Position der feministischen Auseinandersetzung mit Marx als eine „zwischen allen Stühlen“ (ebd.) der Marx-Rezeption. Während der Band von Haubner und Reitz mitunter vor den disziplinären Verengungen soziologischer Debatten nicht gefeit ist, ist die Zusammenstellung der Aufsätze und Themen in diesem Band eher disziplinenübergreifend und interdisziplinär. Dies hat sicher damit zu tun, dass der Anspruch feministischer Forschung und Theoriebildung eben nicht auf eine Bindestrich-Sozialwissenschaft reduziert werden kann, sondern diese Geschlecht als eine Strukturkategorie des Sozialen versteht, die sich in den verschiedenen sozialen Feldern, aber auch quer zu diesen auswirkt.

Die Auseinandersetzung und Kritik des (marxistischen) Feminismus mit den verschiedenen Traditionen marxistischen Denkens hat sich, wie insbesondere die Aufsätze von Frigga Haug, Ingrid Kurz-Scherf oder auch Tina Jung aufzeigen, immer auch als Moment der Fortführung des Marxschen Projektes verstanden. Gleichzeitig ging die Auseinandersetzung des Feminismus mit dem Marxismus über die kritische Revision der Kritik der politischen Ökonomie hinaus und zielte auf eine „Erweiterung seines Analyserahmens, um die zentrale Bedeutung der Trennung in Produktions- und Reproduktionsarbeit für die geschlechtsspezifische Vergesellschaftung“ (9) in den gesellschaftskritischen Auseinandersetzun- 
gen zu verankern. Dabei standen feministische Auseinandersetzungen mit dem marx(isti)schen Denken in einem oft spannungsgeladenen Verhältnis zu den überkommenen Linien marxistischer Theoriebildung, die ihrerseits die feministischen Ansätze oft allzu leichtfertig als „Nebenwiderspruch“ abtaten. Dies hatte sicher nicht zuletzt damit zu tun, dass Marx und Engels sich nicht systematisch mit den Geschlechterverhältnissen auseinandergesetzt hatten, sodass die feministische Auseinandersetzung mit den Werken von Marx (und Engels) vor dem Problem stand, deren Argumentationen überhaupt erst rekonstruieren zu müssen, um kritisch daran anschließen zu können. Die Kritik, dass die Marxschen Begrifflichkeiten zur Untersuchung der Mehrwertproduktion und Ausbeutung eine adäquate Analyse der Geschlechterverhältnisse zumindest erschweren, wenn nicht gar verstellen, konnte von jenen Passagen, in denen Marx und Engels die Relevanz der Geschlechterverhältnisse für den Kapitalismus und die Emanzipation der Gesellschaft benannten, nicht aufgewogen werden. An diese Auseinandersetzungen wird in einer Reihe von Aufsätzen in diesem Band - neben den bereits genannten etwa auch von Julia Dück und Katharina Hajek, aber auch von Silvia Federici (in Schmidt/Fanelli), die den maskulinistischen Charakter der Marxschen Begrifflichkeiten hervorhebt - erinnert.

Neben der Bezugnahme auf die historische Entwicklung der Auseinandersetzung mit marxistischen Ansätzen in den feministischen Debatten ist die Fokussierung auf das Emanzipationsversprechen (Kurz-Scherf) eine bemerkenswerte Gemeinsamkeit einiger Aufsätze dieses Bandes und eine interessante Differenz zum Band von Haubner und Reitz. Dies kann aber nicht auf ein Spezifikum der feministischen Debatten zur Emanzipation von Frauen reduziert werden, sondern verweist m. E. auf ihren Zusammenhang mit gewissen Traditionen des westlichen Marxismus (Anderson, 1978) und seiner Rezeption um und nach 1968, in der Fragen der individuellen Befreiung eine zentrale Rolle spielten. Diese Bezugnahme reicht von der Darstellung der Entwicklung des eigenen wissenschaftlichen Standpunktes zwischen Marxismus und Feminismus, in der sich bei Frigga Haug die Fortentwicklung wissenschaftlicher Debatten mit subjektiven und biographischen Praktiken der Emanzipation und politischen Kämpfen verbanden, bis zu Versuchen, soziale Emanzipation und die damit verknüpften sozialen Kämpfe (nicht nur) von Frauen als zentrales Erkenntnisinteresse der feministischen Auseinandersetzungen mit marxistischen Debatten sichtbar zu machen (Kurz-Scherf) und auch nach den emanzipatorischen Subjektivierungsweisen zu fragen (Hannah Meißner). Der Aufsatz von Alexandra Scheele wiederum untersucht die Spannungen und Widersprüche in den feministischen Debatten zur Frage, ob die Emanzipation eher auf eine Aufwertung der Haus-, Reproduktions- und Care-Arbeit setzen oder ob eher der Eintritt von Frauen in reguläre Erwerbsarbeit, also ihre Proletarisierung, als Voraussetzung für Emanzipation forciert werden solle. 
Die Aufsätze dieses Bandes zeigen, dass es den feministischen Debatten, diesbezüglich vergleichbar marxistischen Ansätzen, um die Analyse des Gesamtzusammenhangs moderner Gesellschaften unter Einbeziehung verschiedener sozialer Dynamiken und Achsen der Ungleichheit geht. Damit bewegen sie sich auf einem ähnlichen epistemologischen Terrain wie marxistisch orientierte Gesellschaftsanalysen. Dieses besteht darin, sich nicht nur auf die detaillierten Untersuchungen einzelner sozialer Felder zu begrenzen, sondern diese - trotz oder wegen ihrer relativen Autonomie - auch in den gesellschaftlichen Gesamtzusammenhang einzubetten oder ihre Beziehungen $\mathrm{zu}$ anderen sozialen Feldern miteinzubeziehen. Ingrid Kurz-Scherf weist aber darauf hin, dass der in vielen marxistischen Debatten aus diesem Grunde sichtbar werdende „überlegene Geltungsanspruch“ (68) der Analyse vorherrschender Vergesellschaftungsmuster in den feministischen Debatten nicht nur kritisiert, sondern für die eigene Theoriebildung trotzdem (eher) abgelehnt wird.

Insgesamt ist daher festzuhalten, dass die Spannungen zwischen feministischen und marxistischen Debatten auf die bei letzteren oftmals noch übliche epistemologische Priorisierung des Kapitalverhältnisses, von dem aus alle weiteren gesellschaftlichen Verhältnisse betrachtet werden, zurückführbar sind. Moderne feministische Debatten würden demgegenüber, wie Kurz-Scherf in Anlehnung an Aulenbacher, Riegraf und Völker (2015) argumentiert, grundsätzlich davon ausgehen, dass moderne Gesellschaften aus einer Konstellation von zumindest drei Herrschaftsverhältnissen (Androzentrismus bzw. geschlechtsbasierte Herrschaft; Eurozentrismus bzw. ethnizitätsbestimmte Herrschaft; Kapitalismus bzw. klassenbasierte Herrschaft) (67) bestehen. Zwar würden auch marxistische Ansätze nicht bestreiten, dass es neben den Klassenverhältnissen auch ethnizitäts- und geschlechtsbasierte „Ungleichheits- und Herrschaftsverhältnisse“ gäbe, es bestünde aber kein Konsens darüber, ob es sich dabei um einen „Strukturzusammenhang“ handelte, diese verschiedenen Achsen der Vergesellschaftung also wechselseitig historisch und systematisch konstitutiv seien (ebd.: 67). Selbst wenn diese Einschätzungen nicht auf alle marxistischen Debatten zutreffen und eine allzu absolut gedachte Kluft zwischen marxistischen und (marxistisch-)feministischen Ansätzen nahelegen, anstatt diese auch als Momente eines umfassenden Projektes kritischer Gesellschaftsanalyse und -theorie zu verstehen, so ist damit doch eine notwendige Reflexionslinie für marxistische Ansätze formuliert, hinter die angesichts der historischen Erfahrungen mit dogmatisierten Debattensträngen und ihrer destruktiven Auswirkungen auf emanzipatorische Kämpfe nicht zurückzufallen ist. 


\section{IIIc. Die Aktualisierung marxistischer Theorietraditionen als interdisziplinäres Projekt}

Es ist ein Spezifikum der an Marx orientierten Analysen in den Sozialwissenschaften, $\mathrm{zu}$ fragen, wie Entwicklungen in verschiedenen gesellschaftlichen Bereichen mit ökonomischen Dynamiken im engeren Sinne vermittelt sind. In der hochspezialisierten Trennung der wissenschaftlichen Disziplinen und nicht zuletzt angesichts der Dominanz einer abstrakten und mathematisierten neoklassischen Ökonomie führt dies immer wieder zum Vorwurf eines ideologisierten Dilettantismus gegen Versuche, ökonomische Erkenntnisse und Konzepte in sozialwissenschaftliche Analysen einzubeziehen. Da aber marxistische Theoriebildung in den Sozialwissenschaften wesentlich auf inter- und transdisziplinären Debatten fußt, ist eben auch die Rezeption und Auseinandersetzung mit aktuellen marxistischen Debatten in der Ökonomie für die Erneuerung marxistischer Theoriebildung und Forschungsstrategien von zentraler Bedeutung. Der von Rainer Lucas, Reinhard Pfriem und Claus Thomasberger herausgegebene Band „Auf der Suche nach dem Ökonomischen - Karl Marx zum 200. Geburtstag“ leistet dazu trotz mancher disziplinbedingter Sperrigkeiten (Formelwüsten in manchen Aufsätzen) für sozialwissenschaftliche Rezipient*innen einen interessanten Beitrag. Dies hat damit zu tun, dass es das gemeinsame Thema der in diesem Band versammelten Autoren ist (es gibt nur bei einem Aufsatz zwei (studentische) Ko-Autorinnen), anhand der Rekonstruktion marxistischer Begrifflichkeiten und Problemstellungen den gesellschaftlichen Charakter der kapitalistischen Ökonomie und ihrer gegenwärtigen Entwicklungen gegen die abstrakte, entgesellschaftlichte Modelllogik neoklassischer Wirtschaftsmodelle herauszuarbeiten. Sie öffnen damit einen epistemologischen Raum für die oben diskutierten sozialwissenschaftlichen Debatten marxistischer und feministischer Provenienz, da sie damit dem „ökonomischen Imperialismus“ erstens den Boden unter den Füßen wegziehen und zweitens die Frage nach der gesellschaftlichen Gestaltbarkeit „des Ökonomischen“ (Thomasberger) öffnen. Durch den gesellschaftstheoretisch fundierten Blick auf das Ökonomische werden die „Objektivationen“ (Thomasberger: 187f.) der neoklassischen Modelle, die ökonomische Dynamiken im Kapitalismus als Naturgesetze erscheinen lassen, als historisch und geographisch spezifische Struktur des „Ökonomischen“ verstehbar, das als von der Gesellschaft getrennt erscheint. Dadurch können auch Probleme wie soziale Ungleichheit und ihre Auswirkungen auf die Gesellschaft auf ökonomische Strukturen bezogen oder die ökologischen Auswirkungen einer auf permanentes Wachstum ausgerichteten Ökonomie in den Blick genommen werden. 
Gleichzeitig wird hier aber eine Begrenzung der Diskussionen dieses Bandes sichtbar, da diese nicht oder nur sehr wenig auf die soziologischen oder feministischen Debatten zu verschiedenen Feldern Bezug nehmen. Eine derartige Herangehensweise hätte aber ihre gesellschaftstheoretische Fundierung stärken und den Blick auf die gesellschaftlichen Macht- und Herrschaftsverhältnisse vertiefen können. Es ist trotzdem eine Stärke einiger Aufsätze dieses Bandes, explizit die Frage nach den Perspektiven für eine nachkapitalistische Gesellschaft und der damit verbundenen Transformation des Verhältnisses zwischen Gesellschaft und Ökonomie aufzuwerfen. Dies ist aber, wie Thomasberger zeigt, ein höchst voraussetzungsvolles Problem, das Marx zwar erkannt, in seinen Vorstellungen zur Veränderung der Gesellschaft jedoch nicht umfassend diskutiert habe. Das „ökonomische Problem“, so Thomasberger, besteht demnach in der Frage, wie die „Integration der verschiedenen wirtschaftlichen Aktivitäten“ und die dafür notwendigen „Entscheidungen über Produktion, Verteilung und Verwendung“ (Thomasberger: 201) getroffen werden. „Wer entscheidet? Auf Basis welcher Informationen? Und nach welchen Gesichtspunkten“ (ebd.).

Dieses Problem werde aber, so Thomasberger weiter, unter kapitalistischen Bedingungen auf ein Informations- und Koordinationsproblem reduziert, das die Lösung über Wettbewerb voraussetzt (ebd.). Marx habe demgegenüber herausgearbeitet, dass eine „grundlegende sozioökologische Transformation der Gesellschaft nur erfolgreich sein kann, wenn das ökonomische Problem in einer komplexen Gesellschaft durch eine neue Form der ,Übereinkunft‘ gelöst wird“ (Thomasberger: 217). Thomasberger bleibt insgesamt zwar eine konkrete Antwort schuldig, er betont aber, dass es verschiedene Antworten geben kann. Der zentrale Punkt seiner Argumentation besteht sicher darin, die Auseinandersetzung mit Marx nochmal auf die Frage zu beziehen, wie eine bewusste, vernünftige und gesellschaftliche Gestaltung des „ökonomischen Problems“ in komplexen Gesellschaften jenseits des Marktes aussehen könnte.

\section{Die Analyse aktueller Entwicklungen}

Die gegenwärtige Relevanz Marxscher Theorien und der daran anknüpfenden Debatten muss sich auch in der Analyse aktueller gesellschaftlicher Probleme erweisen. Exemplarisch diskutieren lässt sich dieses Potenzial an Versuchen, den gegenwärtigen Aufschwung rechtspopulistischer und rechtsextremer Parteien und Bewegungen wie auch ihr Handeln in Regierungspositionen mit der sogenannten „Bonapartismustheorie“ zu erfassen. Diese geht auf die Marxschen Untersuchungen zur Entwicklung in Frankreich nach der Revolution 1848 zurück, die in der Schrift „Der achtzehnte Brumaire des Louis Bonaparte“ kulminierten. Der von 
Martin Beck und Ingo Stützle herausgegebene Band „Die neuen Bonapartisten“ versammelt neben einer Reihe von Aufsätzen (etwa von Hauke Brunkhorst, Dorothea Schmidt, Bob Jessop und Frank Deppe) zu den theoretischen Grundlagen des Bonapartismuskonzeptes in den Schriften von Marx (und Engels) einige Arbeiten (durchaus unterschiedlicher Tiefe), die - mehr oder weniger erfolgreich - versuchen, dieses Konzept zur Analyse politischer Rechtsentwicklungen heranzuziehen.

Der achtzehnte Brumaire gilt als ein Schlüsselwerk der Marxschen Theoriearbeit (siehe Beck und Stützle, Brunkhorst), in dem erstens nachvollziehbar werde, wie Marx abstrakte theoretische Konzepte (z.B. den Klassenbegriff) für die Untersuchung konkreter historischer Situationen fruchtbar machte. Marx untersuchte hier nämlich die Machtübernahme Napoleons III., der 1851 durch einen Staatsstreich diktatorische Vollmachten an sich riss und sich schließlich zum Kaiser des Zweiten Kaiserreichs ausrufen ließ, und wie diese Prozesse mit der spezifischen Klassenstruktur im Frankreich dieser Zeit zusammenhingen. Darüber hinaus entwickelte er zweitens Konzeptionen, die als Wegbereiter einer sozialwissenschaftlichen Revolutionstheorie sowie einer marxistischen Verfassungs- und Demokratietheorie angesehen werden können (siehe Brunkhorst und Stützle). Wie Brunkhorst betont, gehen die Überlegungen von Marx dabei weit über das sehr reduzierte Verständnis des Parlamentarismus und der Demokratie hinaus, das diesbezüglich lange Zeit die marxistischen Debatten beeinträchtigte. Aus der Perspektive der Untersuchung rechtspopulistischer Bewegungen und Regierungen ist drittens interessant, dass Marx die Machtübernahme Napoleons III. als Ergebnis eines Kräftegleichgewichts zwischen den Klassen interpretierte - insbesondere zwischen der Bourgeoisie, die kein tragfähiges Regierungsprojekt entwickeln konnte und der (fragmentierten) bäuerlichen Bevölkerung (Parzellenbauern), die ihre politischen Hoffnungen jedoch auf Napoleon III. projizierte sowie dem sogenannten (v.a. städtischen) Lumpenproletariat (kritische Einwände zu dieser Analyse bringt der Aufsatz von Dorothea Schmidt: 38f.). Durch dieses Kräftegleichgewicht kam es zu einer Autonomisierung des Staates, wodurch der Einfluss des Bürgertums versperrt wurde und die Exekutive schließlich das Parlament entmachtete und eine diktatorische Regierung errichtete. Bereits in den 1930er Jahren hatten marxistische Theoretiker (z.B. Leo Trotzky, August Thalheimer, Otto Bauer) versucht, mit Hilfe der Bonapartismustheorie die Etablierung faschistischer Regime (aber auch des Stalinismus) zu verstehen. Auch in den Debatten zur marxistischen Interpretation des Faschismus nach 1945 wurden diese Überlegungen rezipiert (Saage, 2008). Es ist daher nachvollziehbar, dass der Aufschwung rechtspopulistischer Parteien und ihre Übernahme von Regierungsverantwortung in verschiedenen Ländern ein Phänomen darstellt, anhand dessen die aktuelle Tragfähigkeit des Bonapartismuskonzeptes überprüft werden kann. 
Die Aufsätze, so sie vertieft auf das Bonapartismuskonzept Bezug nehmen, arbeiten heraus, dass eine vereinfachte Analogiebildung zur Situation in Frankreich nach 1848 oder auch zur Durchsetzung des Faschismus zu vermeiden ist. Insbesondere die These des Kräftegleichgewichts als Voraussetzung einer diktatorischen Entwicklung in bürgerlichen Staaten wird in den Aufsätzen bezweifelt, da schon Analysen zur Durchsetzung des Faschismus in den 1930er Jahren gezeigt haben, dass dies eher auf die Schwäche der demokratischen und sozialistischen Kräfte zurückzuführen war. Auch aus heutiger Perspektive sei es angesichts des Machtzugewinns des Kapitals durch neoliberale Regierungsprojekte in den letzten Jahrzehnten schwierig, von einem Kräftegleichgewicht zu sprechen, das durch eine autoritäre Lösung überwunden werden müsste. Insgesamt zeigen die Aufsätze, dass mit der kritischen Rezeption der Marxschen Bonapartismustheorie zur Analyse rechtspopulistischer Bewegungen und Regierungen beigetragen werden kann, da sie die Frage nach Zusammenhängen zwischen politischen Kräfteverhältnissen, Verschiebungen in der Struktur der staatlichen Apparate und der politischen Soziologie einer Gesellschaft und den damit verbundenen ideologischen Formationen aufwirft und allzu einfache Kausalitätsannahmen zur Funktion des Staates oder zur Formierung von Klasseninteressen zu überwinden hilft.

\section{Fazit}

Dieser kurze Überblick über eine Reihe von Publikationen, die sich mit Marx und den an ihm orientierten Theorietraditionen auseinandersetzen, hat gezeigt, dass die vordergründige Renaissance marxistischer Theorien und Konzepte die sozialwissenschaftlichen Auseinandersetzungen vor eine Reihe von Herausforderungen stellt. Der oft behauptete Bedeutungsgewinn Marxscher Theorien kann nämlich allzu leicht darüber hinwegtäuschen, welche Probleme und Schwierigkeiten sich den gesellschaftskritischen, an Marx orientierten Debatten in den Sozialwissenschaften gegenwärtig stellen und mit denen sich aktuelle Auseinandersetzungen konfrontieren müssen. Diese lassen sich unter folgenden Punkten zusammenfassen. Die an marxistischen Theorien und Konzepten orientierten gesellschaftskritischen Diskussionen und Forschungsstrategien in den Sozialwissenschaften waren spätestens seit den 1990er Jahren mit einem massiven Bedeutungsverlust konfrontiert, der nicht nur die im engeren Sinne marxistischen Debatten und Forschungsmöglichkeiten an den Universitäten erfasste, sondern auch ihre Bedeutung für die gesellschaftspolitischen Auseinandersetzungen. Es ist hier nicht der Ort, den gesellschaftlichen Gründen für diese Entwicklungen an den Hochschulen nachzugehen, offensichtlich ist aber, dass ihnen das Zerreißen des (von den marxistischen Ansätzen beanspruchten) Zusammenhanges zwischen der Erarbeitung einer um- 
fassenden Gesellschaftskritik und den sozialen Praxen und Vorstellungen transformativer Akteur`innen zugrunde liegt. Das Praxisproblem der marxistischen Debatten ist auch ein Theorieproblem, wie Kurz-Scherf (in Scheele/Wöhl) prägnant feststellt. Dies hat auch den „Realitätsgehalt“ und „-anspruch“ jener wissenschaftlichen, aber auch politischen Debatten, die beanspruchen, dass eine ,andere Welt möglich sei“, prekär und den „Kapitalismus ohne Alternative“ (Hirsch, 1990) zur scheinbar unhintergehbaren Tatsache werden lassen.

Daraus resultiert die Anforderung an eine erneuerte marxistische Theoriebildung, Begriffe und Konzepte $\mathrm{zu}$ entwickeln, die einerseits die Untersuchung verschiedener gesellschaftlicher Bereiche und Vergesellschaftungsdimensionen ermöglichen, deren Beziehung zu den kapitalistischen Produktionsverhältnissen eine je spezifische Autonomie konstituiert, sodass ihre Funktionsweisen und Dynamiken nicht auf diese zurückgeführt werden können, auch wenn sie von ihnen überformt werden bzw. umgekehrt auf sie auf mannigfaltige Weise zurückwirken. Andererseits erfordert die Erneuerung der marxistischen Theoriebildung und Wissenschaft ihre Öffnung und Artikulation mit feministischen und anti-rassistischen Ansätzen bzw. mit Debatten aus dem globalen Süden und den damit verbundenen sozialen Konfliktfeldern und Emanzipationsprozessen. Die Vielzahl der Bestimmungen im komplexen Ganzen kapitalistischer Gesellschaftsformationen verweist daher auch auf die Vielfalt der sozialen Auseinandersetzungen und Emanzipationsbestrebungen, die die Priorisierung einer Widerspruchsachse, eines Akteurs und einer Praxisform ausschließen sollte.

\section{Literatur}

Althusser, L.; Balibar, É.; Establet, R.; Macherey, P.; Rancière, J., Hrsg. Das Kapital lesen. Vollständigte und ergänzte Ausgabe mit Retraktationen zum Kapital; Westfälisches Dampfboot: Münster, 2015.

Anderson, P. Über den westlichen Marxismus; Syndikat: Frankfurt a. M., 1978.

Atzmüller, R. Marxistische Theoriearbeit in der Krise. Österreichische Zeitschrift für Soziologie 2018, 43, 283-299.

Aulenbacher, B.; Riegraf, B.; Völker, S. Feministische Kapitalismuskritik. Einstiege in bedeutende Forschungsfelder; Westfälisches Dampfboot: Münster, 2015.

Demirović, A., Hrsg. Komplexität und Emanzipation. Kritische Gesellschaftstheorie und die Herausforderung der Systemtheorie Niklas Luhmanns; Westfälisches Dampfboot: Münster, 2001.

Demirovic, A.; Klauke, S.; Schneider, É., Hrsg. Was ist der „Stand des Marxismus“? Soziale und epistemologische Bedingungen der kritischen Theorie heute; Westfälisches Dampfboot: Münster, 2015.

Hardt, M.; Negri, A. Empire; Harvard University Press: Cambridge, Mass (u. a.), 2000.

Hirsch, J. Kapitalismus ohne Alternative. Materialistische Gesellschaftstheorie und Möglichkeiten einer sozialistischen Politik heute; VSA: Hamburg, 1990. 
Marx, K. Das Kapital. Marx-Engels-Werke, Band 23; Dietz: Berlin, 1972.

Marx, K.; Engels, F. Das Manifest der Kommunistischen Partei. Marx-Engels-Werke, Band 4; Dietz: Berlin, 1959a.

Marx, K.; Engels, F. Thesen über Feuerbach. Marx-Engels-Werke, Bd. 3; Dietz: Berlin, 1959b.

Saage, R. Faschismustheorien. Ihre Bedeutung für die Forschung und die politische Bildung. Utopie kreativ 2008, 215, 773-784. 